PROCEEDINGS OF THE

AMERICAN MATHEMATICAL SOCIETY

Volume 131, Number 7 , Pages 2133-2136

S 0002-9939(02)06844-2

Article electronically published on December 30, 2002

\title{
ON STRONG CONVERGENCE TO COMMON FIXED POINTS OF NONEXPANSIVE SEMIGROUPS IN HILBERT SPACES
}

\author{
TOMONARI SUZUKI
}

(Communicated by Jonathan M. Borwein)

\begin{abstract}
In this paper, we prove the following strong convergence theorem: Let $C$ be a closed convex subset of a Hilbert space $H$. Let $\{T(t): t \geq 0\}$ be a strongly continuous semigroup of nonexpansive mappings on $C$ such that $\bigcap_{t>0} F(T(t)) \neq \emptyset$. Let $\left\{\alpha_{n}\right\}$ and $\left\{t_{n}\right\}$ be sequences of real numbers satisfying $0<\alpha_{n}<1, t_{n}>0$ and $\lim _{n} t_{n}=\lim _{n} \alpha_{n} / t_{n}=0$. Fix $u \in C$ and define a sequence $\left\{u_{n}\right\}$ in $C$ by $u_{n}=\left(1-\alpha_{n}\right) T\left(t_{n}\right) u_{n}+\alpha_{n} u$ for $n \in \mathbb{N}$. Then $\left\{u_{n}\right\}$ converges strongly to the element of $\bigcap_{t \geq 0} F(T(t))$ nearest to $u$.
\end{abstract}

\section{INTRODUCTION}

Throughout this paper, we denote by $\mathbb{N}, \mathbb{Q}, \mathbb{R}$ and $\mathbb{R}_{+}$the sets of positive integers, rational numbers, real numbers and nonnegative real numbers, respectively. Let $C$ be a closed convex subset of a Hilbert space $H$. A mapping $T$ on $C$ is called nonexpansive if $\|T x-T y\| \leq\|x-y\|$ for all $x, y \in C$. We denote by $F(T)$ the set of fixed points of $T$. We know that $F(T)$ is nonempty if $C$ is bounded; see 11. Fix $u \in C$. Then for each $\alpha$ with $(0,1)$, there exists a unique point $x_{\alpha}$ of $C$ satisfying $x_{\alpha}=(1-\alpha) T x_{\alpha}+\alpha u$ because the mapping $x \mapsto(1-\alpha) T x+\alpha u$ is contractive. In 1967, Browder 3 proved the following:

Theorem 1 (Browder [3]). Let $C$ be a closed convex subset of a Hilbert space $H$ and let $T$ be a nonexpansive mapping on $C$ with a fixed point. Let $\left\{\alpha_{n}\right\}$ be a sequence of $(0,1)$ converging to 0 . Fix $u \in C$ and define a sequence $\left\{u_{n}\right\}$ by

$$
u_{n}=\left(1-\alpha_{n}\right) T u_{n}+\alpha_{n} u
$$

for $n \in \mathbb{N}$. Then $\left\{u_{n}\right\}$ converges strongly to the element of $F(T)$ nearest to $u$.

Let $\left\{T(t): t \in \mathbb{R}_{+}\right\}$be a strongly continuous semigroup of nonexpansive mappings on a closed convex subset $C$ of a Hilbert space $H$, i.e.,

(1) for each $t \in \mathbb{R}_{+}, T(t)$ is a nonexpansive mapping on $C$;

(2) $T(0) x=x$ for all $x \in C$;

(3) $T(s+t)=T(s) \circ T(t)$ for all $s, t \in \mathbb{R}_{+}$;

(4) for each $x \in X$, the mapping $T(\cdot) x$ from $\mathbb{R}_{+}$into $C$ is continuous.

We put $F(\mathcal{T})=\bigcap_{t \in \mathbb{R}_{+}} F(T(t))$. We know that $F(\mathcal{T})$ is nonempty if $C$ is bounded; see [2]. The following theorem is a corollary of Theorem 8 in [9].

Received by the editors April 14, 2000 and, in revised form, February 12, 2002.

2000 Mathematics Subject Classification. Primary 47H20; Secondary 47H10.

Key words and phrases. Fixed point, nonexpansive semigroup.

(C)2002 American Mathematical Society 
Theorem 2 (Shioji and Takahashi [9]). Let $C$ be a closed convex subset of a Hilbert space $H$. Let $\left\{T(t): t \in \mathbb{R}_{+}\right\}$be a strongly continuous semigroup of nonexpansive mappings on $C$ such that $F(\mathcal{T}) \neq \emptyset$. Let $\left\{\alpha_{n}\right\}$ and $\left\{t_{n}\right\}$ be sequences of real numbers satisfying $0<\alpha_{n}<1, \lim _{n} \alpha_{n}=0, t_{n}>0$ and $\lim _{n} t_{n}=\infty$. Fix $u \in C$ and define a sequence $\left\{u_{n}\right\}$ in $C$ by

$$
u_{n}=\left(1-\alpha_{n}\right) \frac{1}{t_{n}} \int_{0}^{t_{n}} T(s) u_{n} d s+\alpha_{n} u
$$

for $n \in \mathbb{N}$. Then $\left\{u_{n}\right\}$ converges strongly to the element of $F(\mathcal{T})$ nearest to $u$.

In this paper, motivated by the above results, we prove another strong convergence theorem for a strongly continuous semigroup of nonexpansive mappings.

\section{MAin Result}

It is well known that all Hilbert spaces satisfy Opial's condition.

Proposition (Opial [5]). Let $H$ be a Hilbert space. If $\left\{x_{n}\right\}$ is a sequence in $H$ and converges weakly to $z_{0} \in H$, then $\liminf _{n}\left\|x_{n}-z_{0}\right\|<\liminf _{n}\left\|x_{n}-z\right\|$ for all $z \in H$ with $z \neq z_{0}$.

Now we prove our main result.

Theorem 3. Let $C$ be a closed convex subset of a Hilbert space H. Let $\{T(t): t \in$ $\left.\mathbb{R}_{+}\right\}$be a strongly continuous semigroup of nonexpansive mappings on $C$ such that $F(\mathcal{T}) \neq \emptyset$. Let $\left\{\alpha_{n}\right\}$ and $\left\{t_{n}\right\}$ be sequences of real numbers satisfying $0<\alpha_{n}<1$, $t_{n}>0$ and $\lim _{n} t_{n}=\lim _{n} \alpha_{n} / t_{n}=0$. Fix $u \in C$ and define a sequence $\left\{u_{n}\right\}$ in $C$ by

$$
u_{n}=\left(1-\alpha_{n}\right) T\left(t_{n}\right) u_{n}+\alpha_{n} u
$$

for $n \in \mathbb{N}$. Then $\left\{u_{n}\right\}$ converges strongly to the element of $F(\mathcal{T})$ nearest to $u$.

Proof. Let $v$ be the element of $F(\mathcal{T})$ nearest to $u$. From

$$
\begin{aligned}
\left\|u_{n}-v\right\| & =\left\|\left(1-\alpha_{n}\right) T\left(t_{n}\right) u_{n}+\alpha_{n} u-v\right\| \\
& \leq\left(1-\alpha_{n}\right)\left\|T\left(t_{n}\right) u_{n}-v\right\|+\alpha_{n}\|u-v\| \\
& \leq\left(1-\alpha_{n}\right)\left\|u_{n}-v\right\|+\alpha_{n}\|u-v\|
\end{aligned}
$$

we have $\left\|T\left(t_{n}\right) u_{n}-v\right\| \leq\left\|u_{n}-v\right\| \leq\|u-v\|$ for $n \in \mathbb{N}$. Therefore $\left\{u_{n}\right\}$ and $\left\{T\left(t_{n}\right) u_{n}\right\}$ are bounded. Let $\left\{u_{n_{i}}\right\}$ be an arbitrary subsequence of $\left\{u_{n}\right\}$. Then there exists a subsequence $\left\{u_{n_{i_{j}}}\right\}$ of $\left\{u_{n_{i}}\right\}$ which converges weakly to $x$. We claim that $x \in F(\mathcal{T})$. Put $x_{j}=u_{n_{i_{j}}}, \beta_{j}=\alpha_{n_{i_{j}}}$ and $s_{j}=t_{n_{i_{j}}}$ for $j \in \mathbb{N}$. Fix $t>0$. From

$$
\begin{aligned}
\left\|x_{j}-T(t) x\right\| \leq & \sum_{k=0}^{\left[t / s_{j}\right]-1}\left\|T\left((k+1) s_{j}\right) x_{j}-T\left(k s_{j}\right) x_{j}\right\| \\
& +\left\|T\left(\left[t / s_{j}\right] s_{j}\right) x_{j}-T\left(\left[t / s_{j}\right] s_{j}\right) x\right\|+\left\|T\left(\left[t / s_{j}\right] s_{j}\right) x-T(t) x\right\| \\
\leq & {\left[t / s_{j}\right]\left\|T\left(s_{j}\right) x_{j}-x_{j}\right\|+\left\|x_{j}-x\right\|+\left\|T\left(t-\left[t / s_{j}\right] s_{j}\right) x-x\right\| } \\
= & {\left[t / s_{j}\right] \beta_{j}\left\|T\left(s_{j}\right) x_{j}-u\right\|+\left\|x_{j}-x\right\|+\left\|T\left(t-\left[t / s_{j}\right] s_{j}\right) x-x\right\| } \\
\leq & t \beta_{j} / s_{j}\left\|T\left(s_{j}\right) x_{j}-u\right\|+\left\|x_{j}-x\right\|+\max \left\{\|T(s) x-x\|: 0 \leq s \leq s_{j}\right\}
\end{aligned}
$$


for $j \in \mathbb{N}$, we have

$$
\liminf _{j \rightarrow \infty}\left\|x_{j}-T(t) x\right\| \leq \liminf _{j \rightarrow \infty}\left\|x_{j}-x\right\| .
$$

By the Proposition, this implies $T(t) x=x$. Therefore $x \in F(\mathcal{T})$. We next prove $\left\{x_{j}\right\}$ converges strongly to $v$. From

$$
\begin{aligned}
& \beta_{j}\left\|x_{j}-v\right\|^{2}+\left(1-\beta_{j}\right)\left\langle\left(x_{j}-T\left(s_{j}\right) x_{j}\right)-\left(v-T\left(s_{j}\right) v\right), x_{j}-v\right\rangle \\
& =\beta_{j}\left\langle u-v, x_{j}-v\right\rangle
\end{aligned}
$$

and

$$
\begin{aligned}
& \left\langle\left(x_{j}-T\left(s_{j}\right) x_{j}\right)-\left(v-T\left(s_{j}\right) v\right), x_{j}-v\right\rangle \\
& \geq\left\|x_{j}-v\right\|^{2}-\left\|T\left(s_{j}\right) x_{j}-T\left(s_{j}\right) v\right\| \cdot\left\|x_{j}-v\right\| \\
& \geq 0
\end{aligned}
$$

we obtain $\left\|x_{j}-v\right\|^{2} \leq\left\langle u-v, x_{j}-v\right\rangle$ for $j \in \mathbb{N}$. Since $\langle u-v, x-v\rangle \leq 0$, we have

$$
\begin{aligned}
\left\|x_{j}-v\right\|^{2} & \leq\left\langle u-v, x_{j}-v\right\rangle \\
& =\left\langle u-v, x_{j}-x\right\rangle+\langle u-v, x-v\rangle \\
& \leq\left\langle u-v, x_{j}-x\right\rangle
\end{aligned}
$$

for $j \in \mathbb{N}$ and hence $\left\{x_{j}\right\}$ converges strongly to $v$. Since $\left\{u_{n_{i}}\right\}$ is arbitrary, we obtain that $\left\{u_{n}\right\}$ converges strongly to $v$.

We have some remarks about Theorem [3.

Remark. (1) By the proof of theorem 5.1 in 7 , we can prove the following statement: Let $E$ be a smooth uniformly convex Banach space with a duality mapping which is weakly sequentially continuous at zero, and let $C$ be a closed convex subset of $E$. Let $\left\{T(t): t \in \mathbb{R}_{+}\right\},\left\{\alpha_{n}\right\},\left\{t_{n}\right\}, u$ and $\left\{u_{n}\right\}$ be as in Theorem 3 , Then $\left\{u_{n}\right\}$ converges strongly to $P u$, where $P$ is the sunny nonexpansive retract from $C$ onto $F(\mathcal{T})$.

(2) Halpern 4 proved the strong convergence theorem for a nonexpansive mapping by the explicit iteration. So, we have one problem of whether there is an explicit iteration concerning Theorem 3 .

\section{APPENDIX}

In Theorem 3, it is needed that $T(\cdot) x$ is continuous for all $x \in C$. In this section, we give an example. By Axiom of Choice, there exist a subset $\mathbb{A}$ of $\mathbb{R}_{+}$and a mapping $\theta$ from $\mathbb{R}_{+}$onto $\mathbb{A}$ such that $\theta^{-1}(a)=(a+\mathbb{Q}) \cap \mathbb{R}_{+}$for all $a \in \mathbb{A}$. Note that the following hold:

(1) $\theta(a)=a$ for all $a \in \mathbb{A}$;

(2) $\theta(t)-t \in \mathbb{Q}$ for all $t \in \mathbb{R}_{+}$;

(3) $\theta(s)=\theta(t)$ if and only if $s-t \in \mathbb{Q}$ for all $s, t \in \mathbb{R}_{+}$;

(4) $\theta(t+q)=\theta(t)$ and $\theta(\theta(s)+t)=\theta(s+t)$ for all $q \in \mathbb{Q} \cap \mathbb{R}_{+}$and $s, t \in \mathbb{R}_{+}$.

Example. Let $H$ be a Hilbert space consisting of all the functions $x$ from $\mathbb{A}$ into $\mathbb{R}$ satisfying $\sum_{a \in \mathbb{A}}|x(a)|^{2}<\infty$ with inner product $\langle x, y\rangle=\sum_{a \in \mathbb{A}} x(a) \cdot y(a)$ for all $x, y \in H$. Define a semigroup $\left\{T(t): t \in \mathbb{R}_{+}\right\}$of linear nonexpansive mappings on $H$ by $(T(t) x)(a)=x(\theta(a+t))$ for all $x \in H$ and $a \in \mathbb{A}$. Fix $u \in H$ satisfying 
$u(\theta(0))=1$ and $u(a)=0$ for all $a \in \mathbb{A}$ with $a \neq \theta(0)$. Define a sequence $\left\{u_{n}\right\}$ in $H$ by

$$
u_{n}=\left(1-1 / n^{2}\right) T(1 / n) u_{n}+\left(1 / n^{2}\right) u
$$

for $n \in \mathbb{N}$. Then $u_{n}=u$ for $n \in \mathbb{N}$ and $u$ is not a common fixed point of $\{T(t): t \in$ $\left.\mathbb{R}_{+}\right\}$.

Proof. We first show that the mapping $\tau_{t}$ on $\mathbb{A}$ defined by $\tau_{t}(a)=\theta(a+t)$ is bijective for each $t \in \mathbb{R}_{+}$. If $\theta(a+t)=\theta(b+t)$ for some $a, b \in \mathbb{A}$, then $(a+t)-(b+t)=$ $a-b \in \mathbb{Q}$. So, we obtain $a=\theta(a)=\theta(b)=b$. For each $a \in \mathbb{A}$, we have

$$
\tau_{t}(\theta(a-t+[t]+1))=\theta(\theta(a-t+[t]+1)+t)=\theta(a+[t]+1)=\theta(a)=a .
$$

These imply that $\tau_{t}$ is bijective for each $t \in \mathbb{R}_{+}$. Hence, $T(t)$ is well-defined and isometric for each $t \in \mathbb{R}_{+}$. Note that $F(\mathcal{T})=\{0\}$. Since

$$
\begin{aligned}
(T(s) \circ T(t) x)(a) & =(T(t) x)(\theta(a+s))=x(\theta(\theta(a+s)+t)) \\
& =x(\theta(a+s+t))=(T(s+t) x)(a)
\end{aligned}
$$

for $x \in H$ and $a \in \mathbb{A}$, we have $T(s+t)=T(s) \circ T(t)$ for all $s, t \in \mathbb{R}_{+}$. This shows that $\left\{T(t): t \in \mathbb{R}_{+}\right\}$is a semigroup. From $(T(q) x)(a)=x(\theta(a+q))=x(a)$ for

$a \in \mathbb{A}$, we have $T(q) x=x$ for all $q \in \mathbb{Q} \cap \mathbb{R}_{+}$and $x \in H$. Especially, $T(0) x=x$ for all $x \in H$. However, for each $x \in H$ with $x \neq 0, T(\cdot) x$ is not continuous everywhere. Since $T(1 / n) u_{n}=u_{n}$, we get $u_{n}=u$ for $n \in \mathbb{N}$. This completes the proof.

\section{ACKNOWLEDGMENT}

The author wishes to express his sincere thanks to the referee for giving valuable suggestions.

\section{REFERENCES}

1. F. E. Browder, "Fixed-point theorems for noncompact mappings in Hilbert space", Proc. Nat. Acad. Sci. USA, 53 (1965), 1272-1276. MR 31:2582

2. F. E. Browder, "Nonexpansive nonlinear operators in a Banach space", Proc. Nat. Acad. Sci. USA, 54 (1965), 1041-1044. MR 32:4574

3. F. E. Browder, "Convergence of approximates to fixed points of nonexpansive nonlinear mappings in Banach spaces", Arch. Ration. Mech. Anal., 24 (1967), 82-90. MR 34:6582

4. B. Halpern, "Fixed points of nonexpanding maps", Bull. Amer. Math. Soc., 73 (1967), 957-961. MR 36:2022

5. Z. Opial, "Weak convergence of the sequence of successive approximations for nonexpansive mappings", Bull. Amer. Math. Soc., 73 (1967), 591-597. MR 35:2183

6. S. Reich, "Approximating zeros of accretive operators", Proc. Amer. Math. Soc., 51 (1975), 381-384. MR 57:10508

7. S. Reich, "Extension problems for accretive sets in Banach spaces", J. Funct. Anal., 26 (1977), 378-395. MR 57:17393

8. S. Reich, "Strong convergence theorems for resolvents of accretive operators in Banach spaces", J. Math. Anal. Appl., 75 (1980), 287-292. MR 82a:47050

9. N. Shioji and W. Takahashi, "Strong convergence theorems for asymptotically nonexpansive semigroups in Hilbert spaces", Nonlinear Anal., 34 (1998), 87-99. MR 99e:47071

Department of Mathematics and Information Science, Graduate School of Science and Technology, Nimgata University, Nitgata 950-2181, Japan

E-mail address: tomonari@math.sc.niigata-u.ac.jp 